\title{
Combined Flocking and Distance-Based Shape Control of Multi-Agent Formations
}

\author{
Mohammad Deghat, Member, IEEE, Brian D.O. Anderson, Life Fellow, IEEE, and \\ Zhiyun Lin, Senior Member, IEEE
}

\begin{abstract}
Steepest descent control laws can be used for formation shape control based on specified inter-agent distances, assuming point agents with single integration of the control action to yield velocity. Separately, it is known how to achieve equal velocity for a collection of agents in a formation using consensus ideas, given appropriate properties for the graph describing information flows. This work shows how the two concepts of formation shape control and flocking behavior can be combined when one changes from an agent with single integration to one with double integration.
\end{abstract}

\section{INTRODUCTION}

In recent years, there has been much attention given to the control of formations of multiple agents to achieve certain objectives. The class of formation control problems that have been considered includes formation shape control, where the formation's agents are controlled so that the formation takes up a particular shape whose orientation and center of mass position are irrelevant.

Most of the literature assumes point agents operating in $\mathbb{R}^{2}$ and we shall do that too. Shape control under these circumstances proceeds from a nominated set of inter-agent distances $d_{i j}^{*}, i j \in \overline{\mathcal{E}}$, where $\overline{\mathcal{E}}$ refers to the edge set of a graph $\overline{\mathcal{G}}=(\overline{\mathcal{V}}, \overline{\mathcal{E}})$. The set $\overline{\mathcal{V}}$ denotes the vertices, which correspond to the individual agents. When agents $i, j$ at the end of an edge ij are aware of its current and desired length, there exists a gradient based control law which moves the formation to an equilibrium; each agent uses the relative positions of its neighbors (two agents being neighbors if the corresponding vertices of the graph are adjacent), and the desired distances to its neighbors. This enables construction of a distributed control law, which expresses the agent velocity in terms of the measurement data available to the agent in question, and the desired distances. Further, the agents do not need to share a common coordinate basis. A comprehensive treatment can be found in e.g. [2].

Mohammad Deghat is with School of Engineering and Information Technology, UNSW-Canberra, ACT 2600 Australia (email: m.deghat@unsw.edu.au).

Brian D.O. Anderson is with Research School of Engineering, Australian National University, Canberra, ACT 0200 and National ICT Australia (email: brian.anderson@anu.edu.au).

Zhiyun Lin is with State Key Laboratory of Industrial Control Technology, College of Electrical Engineering, Zhejiang University, Hangzhou 310027 China (email: linz@zju.edu.cn).

This work is supported by NICTA, which is funded by the Australian Government as represented by the Department of Broadband, Communications and the Digital Economy and the Australian Research Council through the ICT Centre of Excellence program and by ARC grant DP-0877562 and DP 110100538. M. Deghat's work was done while at ANU.

The material in this paper is partially presented as a book chapter in [1].
Another class of formation control problem arises when one wants all agents in a formation to assume a common velocity. This is a consensus problem. Associated with it is an information graph, which in general is directed; there is an edge directed from vertex $i$ to vertex $j$ when agent $i$ 's velocity is available to agent $j$. Of course, it is not precluded that information exchange is reciprocal, and in this case an undirected graph can be used. In the directed case, when the graph has a spanning tree (or equivalently has a unique closed strongly connected subgraph), consensus can be achieved. If one agent has no incoming edges, it is a leader and there are directed paths from it to every agent, all agents acquire its velocity, assuming the graphical condition holds. In the undirected case, the graph simply needs to be connected. When consensus can be achieved with an undirected information graph, and then one agent is designated as a leader, its incident edges are all converted to outwardly directed edges and consensus is again achievable, based on the leader's velocity. These results can be found in e.g. [3] and [4] and are reviewed further below.

Our aim in this paper is to show how one can control a formation so that both objectives of shape control and sharing of a common velocity ${ }^{1}$ are achieved. Note that it does make sense to consider the objectives separately in terms of formation operations. One can well imagine that a formation has assembled in a correct shape and moves from point $\mathrm{A}$ to point $\mathrm{B}$, at which point it turns. To accommodate the turn, one simply uses consensus-with-a-leader, and one would want the shape control aspect to take care of itself. That concept is achieved in our scheme.

This idea has also been considered by Olfati-Saber [5], Tanner et. al. [6], Dimarogonas and Johansson [7] and $\mathrm{Su}$ et. al. [8]. These authors assume the graph for underlying formation shape control and consensus are undirected, and the formation has no leader. Our main contribution is to consider the general case where the graphical structures of the consensus and shape control graphs are not necessarily identical. We further assume different cases where there is a leader or a group of leaders in the formation and the consensus graph is directed. The stability analysis is performed using Lyapunov stability theorem and LaSalle's invariance principle, and it is shown that the system is asymptotically stable. We further show using the center manifold theorem that the correct equilibrium associated with the desired formation shape and

\footnotetext{
${ }^{1}$ The common velocity may be that of a leader, or an average of the
} velocities of all the agents. 
velocity is locally exponentially stable. The idea of using center manifold theory has been used in [2], [9] to show the stability of multi-agent systems, but their analysis is restricted to only shape control problem. We, however, apply center manifold theory to a more general problem, i.e. flocking with shape control.

There are other results in the literature in which the agents in the formation should keep a certain relative position (i.e., both distance and orientation) while moving from point A to point B, see e.g. [10], [11]. A fundamental distinction between the problem considered in [10], [11] and the problem considered here is that [10], [11] study the case where both the shape and orientation of the formation should be maintained, whereas we assume that just the shape of the formation, which can be maintained by controlling inter-agent distances, is important and the orientation is irrelevant. The former problem can be done simply with a linear system, but it requires all agents to agree on a common direction, say e.g. north, while the latter does not.

The material in this paper is partially presented in [1]. The main contributions of this paper, in comparison to [1], are as follows: it considers the case where the consensus graph has a closed strongly connected component which contains more than a single vertex, and includes complete proofs of the results in [1] and the exponential stability proof using the center manifold theorem.

The structure of the paper is as follows. In the next section we define the notation, and provide formal statements of the separate results for shape control and consensus velocity control. Section III indicates how to combine the two ideas, and Section IV contains simulation results. Conclusions and suggestions for future work are presented in Section V.

\section{REVIEW OF BACKGROUND THEORY}

\section{A. Notation}

We consider $n$ point agents in $\mathbb{R}^{2}$, with the $i$-th agent located at $p_{i}$. We denote by $d_{i j}$ the distance between agents $i, j: d_{i j}=$ $\left\|p_{i}-p_{j}\right\|$, and we let $d_{i j}^{*}$ denote a specified or desired distance between the same agents. We denote by $\overline{\mathcal{N}}_{i}$ the neighbor set of agent $i$, or the set of agents $j$ for which $d_{i j}^{*}$ is specified. We let $p$ denote the vector of all agent positions stacked together. Likewise, $q_{i}=\dot{p}_{i}$ will denote the velocity of agent $i$, and $q=\dot{p}$ will denote the vector of all agent velocities stacked together.

For shape control purposes, an underlying undirected graph $\overline{\mathcal{G}}=(\overline{\mathcal{V}}, \overline{\mathcal{E}})$ is specified where $\overline{\mathcal{V}}$ is a list of vertices, one corresponding to each agent, and $\overline{\mathcal{E}}$ is a list of agent pairs (edges of the graph) where it is assumed that $i j \in \overline{\mathcal{E}}$ if and only if $d_{i j}^{*}$ is specified and $d_{i j}(t)$ at any time $t$ is known to agents $i, j$.

\section{B. Shape control}

In most shape control problems, it is usual to postulate that the set of edges $\overline{\mathcal{E}}$ defined by the distance data ensures that the associated graph $\overline{\mathcal{G}}$ is at least rigid, if not globally rigid. Rigidity means that if a formation is such that at some time $t$ there holds $d_{i j}(t)=d_{i j}^{*}$ for all $i j \in \overline{\mathcal{E}}$, then the only continuous motions of the formation are ones which preserve its shape, i.e. when the formation moves, so that the geometric figure after the move must be congruent with that before the move. Global rigidity means that all formations achieving the specified distances are congruent, i.e. differ at most by translation, rotation, or reflection. See [12] for more details. Note that there exists rigid but not globally rigid formations. Consider for example a formation with four agents, and with specification of the lengths of edges $12,23,31,24,41$ as shown in Fig. 1. It is not hard to see that the formation comprises 2 triangles, 123 and 124 with a common base. The triangle shapes are individually determined. However, one may have the two triangles on the same or on opposite sides of the base and be consistent with given distance data. Of course, there is no continuous motion preserving nominated distances and carrying one of these formation shapes into the other. So this formation is rigid, but is not globally rigid.
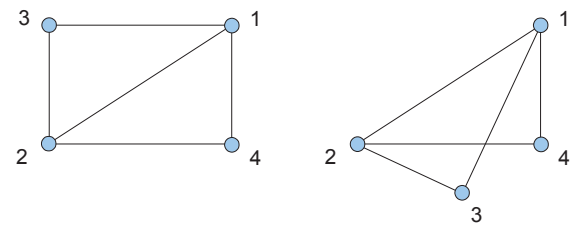

Fig. 1: A framework which is rigid, but not globally rigid.

A framework $(\overline{\mathcal{G}}, p)$ is generically rigid if it is rigid and $p$ is generic. Generic rigidity is a property of the graph $\overline{\mathcal{G}}$, not the configuration $p$. Thus, we also say a graph $\overline{\mathcal{G}}$ is rigid in this paper if the framework $(\overline{\mathcal{G}}, p)$ is generically rigid for any generic configuration $p$ in the plane.

Another important type of rigidity is infinitesimal rigidity which is a stronger condition than rigidity though not as strong as global rigidity. Let the framework $(\overline{\mathcal{G}}, p)$ be defined as

$$
(\overline{\mathcal{G}}, p):=\left\{p_{i}, p_{j} \in \mathbb{R}^{2} \mid\left\|p_{i}-p_{j}\right\|=d_{i j}^{*} \forall\{i, j\} \in \overline{\mathcal{E}}\right\} .
$$

Then the framework $(\overline{\mathcal{G}}, p)$ is infinitesimally rigid if every possible motion that satisfies

$$
\left(q_{i}(t)-q_{j}(t)\right)^{\top}\left(p_{i}(t)-p_{j}(t)\right)=0 \forall\{i, j\} \in \overline{\mathcal{E}}
$$

consists of only rotation and translation of the whole framework [13].

It is valid to attempt to control a formation to a given shape. Typically a function measuring the error between the current formation and the desired formation is specified. One such choice of 'fidelity index' is

$$
V(p)=\frac{1}{2} \sum_{i j \in \overline{\mathcal{E}}}\left(d_{i j}^{2}-d_{i j}^{* 2}\right)^{2} .
$$

Notice that this expression is invariant under translation, rotation or reflection of the formation. Variants on this expression include adjustments to penalize excessive closeness of agents, and excessive separation of agents, reflecting the desire to avoid agent collisions, and to maintain agents in contact with one another, when communication over long distances may fail. See for example [7], [14]. 
The control law used is simply a steepest descent law, thus

$$
\dot{p}=-\nabla V(p) \text {. }
$$

The particular equation for agent $i$ is

$$
\dot{p}_{i}=2 \sum_{j \in \overline{\mathcal{N}}_{i}}\left(d_{i j}^{* 2}-d_{i j}^{2}\right)\left(p_{i}-p_{j}\right) .
$$

As is typical for shape control laws, motion of agent $i$ is defined solely using the agent's own neighbors; their relative positions (in agent $i$ 's coordinate base) are required, as is the distance error. As shown in e.g. [2], convergence of the formation shape always occurs if the required graphical conditions hold. With the particular index above, the center of mass of the formation (i.e. $\sum_{i=1}^{n} p_{i}$ ) remains constant. The question of whether convergence occurs to a correct equilibrium, i.e. one at which the specified distances are attained, has been considered in much detail; for formations of three or more agents, there are always multiple equilibria including the correct equilibria, and the latter are always attractive, so local convergence around a correct equilibrium is guaranteed. The existence of attractive incorrect equilibria can so far only be excluded in a limited number of cases; for example, for a triangular formation, none of the incorrect equilibria, which involve either two or three agents being collocated or three agents being collinear, are attractive. In all cases, equilibria are not isolated: this is because any rotation or translation is also an equilibrium. This fact makes more difficult the convergence analysis of equilibria. Nevertheless, with the aid of the center manifold theory, exponential convergence to zero of $V(p)$ and thus the individual distance error terms such as $\left(d_{i j}^{2}-d_{i j}^{* 2}\right)^{2}$ can be demonstrated in the vicinity of a correct equilibrium.

As noted above, $\sum_{i} p_{i}$ remains constant under the steepest descent law associated with the fidelity index (3). We have the following generalization, to which we will later appeal:

Lemma 1: Let $V(p)$ be a continuously differentiable function of the distance between a subset of agents of a formation. Then on motions defined by $\dot{p}=-\nabla V(p)$, there holds (a) $\left(1 \otimes I_{2}\right)^{\top} \nabla V(p)=0$ for all $p$ or equivalently (b) $\sum_{i} p_{i}$ remains constant. Here 1 denotes the $n$-vector of all 1's.

Proof: Note that $\left(\mathbf{1} \otimes I_{2}\right)^{\top} \nabla V(p)=\sum_{i} \frac{\partial V(p)}{\partial p_{i}}$, and if $d_{i j}$ is the distance between $i$ and $j$ then

$$
\frac{\partial V(p)}{\partial d_{i j}} \frac{\partial d_{i j}}{\partial p_{i}}=-\frac{\partial V(p)}{\partial d_{i j}} \frac{\partial d_{i j}}{\partial p_{j}} .
$$

So it is evident that $\sum_{i} \frac{\partial V(p)}{\partial p_{i}}=\left(\mathbf{1} \otimes I_{2}\right)^{\top} \nabla V(p)=0$. Next, on motions defined by $\dot{p}=-\nabla V(p)$, there must hold $(\mathbf{1} \otimes$ $\left.I_{2}\right)^{\top} \dot{p}=-\left(\mathbf{1} \otimes I_{2}\right)^{\top} \nabla V(p)=0$ which is equivalent to $\sum_{i} p_{i}$ remains constant.

\section{Flocking behavior}

By flocking behavior, we mean the phenomenon that all agents in a formation acquire an identical velocity. Let $\mathcal{G}=$ $(\mathcal{V}, \mathcal{E})$ denote a graph, which may be directed, governing the information transmission between pairs of agents. There is an edge in $\mathcal{E}$ from vertex $i$ to vertex $j$ when agent $j$ can learn agent $i$ 's velocity instantaneously. We say a graph is rooted at vertex $\ell$ if there is a path from $\ell$ to every vertex in the graph.
Let $\mathcal{N}_{i}$ denote the set of agents $j$ supplying information to agent $i$. Let $L$ denote the Laplacian matrix associated with $\mathcal{G}$. Thus for $i \neq j$, there holds $l_{i j}=-1$ if and only if there is an edge from $j$ to $i$; else $l_{i j}=0$. Moreover, $l_{i i}=-\sum_{j} l_{i j}$. Another matrix that interprets the adjacency relationship in a graph is the incidence matrix. The $k$ th column of the incidence matrix $H$ is determined by the $k$ th edge of graph $\mathcal{G}$. If the $k$ th edge is pointing from vertex $i$ to vertex $j$, then the $i$ th entry of column $k$ is -1 and the $j$ th entry is 1 . If $L$ is the Laplacian matrix associated with an undirected graph, then $L$ can be written as $L=H H^{\top}$. According to the definition of $H$, the incidence matrix has each column summing to zero, i.e. $H^{\top} \mathbf{1}=0$.

Define $\mathcal{L}=L \otimes I_{2}$. The consensus equation, defining how each agent adjusts its velocity given the information available to it, is

$$
\dot{q}=-\mathcal{L} q
$$

For agent $i$, this is

$$
\dot{q}_{i}=\sum_{j \in \mathcal{N}_{i}}\left(q_{j}-q_{i}\right)
$$

Then the following holds [3]:

Theorem 1: With notation as above, suppose $\mathcal{G}$ is undirected, and so the associated Laplacian is symmetric. Then the following conditions are equivalent:

1) Equation (6) converges exponentially fast from any initial condition to a solution in which all $q_{i}$ assume the same vector value in $\mathbb{R}^{2}$.

2) $\mathcal{G}$ is connected.

3) The kernel of $L$ is 1 -dimensional, being $\operatorname{span}\{\mathbf{1}\}$, where 1 denotes a vector of all 1's. The cokernel is also $\operatorname{span}\{\mathbf{1}\}$ as $L$ is symmetric for an undirected graph. All nonzero eigenvalues of $L$ are positive.

If $\mathcal{G}$ is directed, condition (1) remains as before, and the other two conditions are replaced by three equivalent conditions:

1) $\mathcal{G}$ has a spanning tree.

2) $\mathcal{G}$ has a unique closed strongly connected component ${ }^{2}$.

3) The kernel of $L$ is 1 -dimensional, being $\operatorname{span}\{\mathbf{1}\}$. The cokernel is spanned by a nonzero vector $\eta$ of all nonnegative entries, and all entries are positive if and only if $\mathcal{G}$ is strongly connected. Nonzero eigenvalues of $L$ have positive real parts.

\section{Center Manifold Theory}

Center manifold theory is a tool for determining the stability of a nonlinear system which has eigenvalues with zero real parts when linearized about an equilibrium point. It is a powerful tool that studies the stability of a nonlinear system by analyzing a lower order nonlinear system whose order is exactly equal to the number of eigenvalues of the linearized system with zero real parts. Standard treatments of center manifold theory can be found in e.g. [15], [16]. We briefly explain the center manifold theory below.

\footnotetext{
${ }^{2}$ A strongly connected component is a maximal strongly connected subgraph. A closed strongly connected component is one such that there are no inwardly directed edges to any vertex in the component from a vertex outside the component.
} 
Consider the system

$$
\begin{aligned}
& \dot{\theta}=A_{c} \theta+h_{1}(\theta, \rho) \\
& \dot{\rho}=A_{s} \rho+h_{2}(\theta, \rho), \quad(\theta, \rho) \in \mathbb{R}^{\kappa} \times \mathbb{R}^{\vartheta-\kappa}
\end{aligned}
$$

where $A_{c}$ is a matrix having eigenvalues with zero real parts, $A_{s}$ is a matrix having eigenvalues with negative real parts, and the functions $h_{1}(\theta, \rho)$ and $h_{2}(\theta, \rho)$ are $C^{2}$ functions satisfying $h_{1}(0,0)=0, h_{2}(0,0)=0, J_{h_{1}}(0,0)=0$ and $J_{h_{2}}(0,0)=0 .{ }^{3}$ An invariant manifold is called a center manifold for (8) if it can be locally represented as

$$
\mathcal{M}:=\left\{(\theta, \rho) \in \mathbb{R}^{\kappa} \times \mathbb{R}^{\vartheta-\kappa}: \rho=h(\theta)\right\}
$$

for some sufficiently small neighborhood of the origin where the function $h(\theta)$ satisfies $h(0)=0$ and $J_{h}(0)=0$.

It can be shown that center manifold exists for (8) with local representation function $h: \mathbb{R}^{\kappa} \rightarrow \mathbb{R}^{\vartheta-\kappa}$. The dynamics of (8) restricted to any such center manifold is given by the following nonlinear system for sufficiently small $\xi$ :

$$
\dot{\xi}=A_{c} \xi+h_{1}(\xi, h(\xi)), \quad \xi \in \mathbb{R}^{\kappa} .
$$

The stability of (8) can be analyzed from the dynamics of the center manifold in (10) using the following theorem.

Theorem 2: (Thm. 7.26 [16]) Consider the system of equations (8) and its associated center manifold system (10). If the origin of (10) is stable (asymptotically stable) (unstable), then the origin of (8) is stable (asymptotically stable) (unstable). Suppose the origin of (10) is stable. Then if $(\theta(t), \rho(t))$ is a solution of (8) starting with a sufficiently small $(\theta(0), \rho(0))$, there is a solution $\xi(t)$ of (10) such that as $t \rightarrow \infty$

$$
\begin{aligned}
\theta(t) & =\xi(t)+O\left(e^{-\gamma t}\right) \\
\rho(t) & =h(\xi(t))+O\left(e^{-\gamma t}\right)
\end{aligned}
$$

where $\gamma$ is a positive constant.

\section{COMBINING SHAPE CONTROL AND FLOCKING}

Throughout this section, $\overline{\mathcal{G}}$ denotes a shape control graph. We consider several possibilities for the consensus graph $\mathcal{G}$.

\section{A. Undirected consensus graph}

We first consider an undirected consensus graph and state the following theorem.

Theorem 3: Suppose there is an undirected consensus graph $\mathcal{G}$ which is connected and an underlying formation shape graph $\overline{\mathcal{G}}$ which is rigid. Let $\mathcal{L}=L \otimes I_{2}$ with $L$ the Laplacian associated with $\mathcal{G}$ and let $V(p)$ be a function invariant to translation, rotation and reflection. Then for the double-integrator model combining shape control and velocity consensus:

$$
\begin{aligned}
\dot{p} & =q \\
\dot{q} & =-\mathcal{L} q-\nabla V(p),
\end{aligned}
$$

all trajectories tend as $t \rightarrow \infty$ to the set

$$
\Omega=\{(p, q): \mathcal{L} q=0 \text { and } \nabla V(p)=0\} .
$$

\footnotetext{
${ }^{3} J_{f}(x)$ denotes the Jacobian of $f(x)$ evaluated at a point $x$.
}

Proof: Our approach will be to work with a Lyapunovlike function, nonnegative but not positive definite in the state, and to show that its time derivative is nonpositive. We then apply the LaSalle's invariance principle, but for this purpose, we need separately to establish that all trajectories are bounded. (Note the applicability of LaSalle in this situation [16]). Since the state trajectory $p(t)$ is evidently not bounded once the formation is moving, a state-variable change is needed.

Observe first that because $\left(\mathbf{1} \otimes I_{2}\right)^{\top} \mathcal{L}=0$, and also because $\left(\mathbf{1} \otimes I_{2}\right)^{\top} \nabla V(p)=0$ in the light of Lemma 1 , there holds $\left(\mathbf{1} \otimes I_{2}\right)^{\top} \dot{q}=0$; equivalently, $\left(\mathbf{1} \otimes I_{2}\right)^{\top} q(t)$ is constant. This simply reflects the fact that the average velocity of all agents stays the same.

Define the transformations as

$$
\begin{aligned}
& \bar{p}(t)=p(t)-\left(\left(\mathbf{1} \otimes I_{2}\right) \frac{\left(\mathbf{1} \otimes I_{2}\right)^{\top} q(t)}{n}\right) t \\
& \bar{q}(t)=q(t)-\left(\mathbf{1} \otimes I_{2}\right) \frac{\left(\mathbf{1} \otimes I_{2}\right)^{\top} q(t)}{n}
\end{aligned}
$$

where $n$ is the number of agents. It is evident from the definition of $\bar{q}(t)$ that $\left(\mathbf{1} \otimes I_{2}\right)^{\top} \bar{q}(t)=0$. This simply reflects the fact that the average velocity of all agents in the new coordinate system is zero. Hence $\left(\mathbf{1} \otimes I_{2}\right)^{\top} \dot{\bar{p}}(t)=0$ or $\left(\mathbf{1} \otimes I_{2}^{\top}\right) \bar{p}(t)$ is constant, which means that in the $\bar{p}(t)$ coordinates, the center of gravity of the agent positions is fixed.

Then the equations of motion in the new coordinates which strip out the average motion of the formation become

$$
\begin{aligned}
\dot{\bar{p}} & =\dot{p}-\left(\mathbf{1} \otimes I_{2}\right) \frac{\left(\mathbf{1} \otimes I_{2}\right)^{\top} q(t)}{n} \\
& =q-\left(\mathbf{1} \otimes I_{2}\right) \frac{\left(\mathbf{1} \otimes I_{2}\right)^{\top} q(t)}{n} \\
& =\bar{q} \\
\dot{\bar{q}} & =-\mathcal{L} \bar{q}-\nabla V(\bar{p}) .
\end{aligned}
$$

Notice that $V(p)=V(\bar{p})$, since $p_{i}(t)-p_{j}(t)=\bar{p}_{i}(t)-\bar{p}_{j}(t)$.

Now take as a Lyapunov function $W(\bar{p}, \bar{q})=(1 / 2) \bar{q}^{\top} \bar{q}+$ $V(\bar{p})$, and verify that $\dot{W}(\bar{p}, \bar{q})=-\bar{q}^{\top} \mathcal{L} \bar{q}$. Since this is nonpositive, it follows that $W$ is bounded and then that $\bar{q}(t)$ is bounded and $V(\bar{p}(t))$ is bounded. Since $V(\bar{p})$ is bounded and the graph $\overline{\mathcal{G}}$ is connected, all the edge lengths $\left\|\bar{p}_{i}(t)-\bar{p}_{j}(t)\right\|$ are bounded. Because the edge lengths are bounded, because $\overline{\mathcal{G}}$ is connected, and because the center of gravity of the formation in the $\bar{p}$-coordinates is constant, $\bar{p}(t)$ itself is bounded.

Because of the boundedness of $\bar{p}(t)$ and $\bar{q}(t)$, the LaSalle's Invariance Principle applies and it guarantees all trajectories converge to the largest invariant set in $S=\{(\bar{p}, \bar{q}): \dot{W}(\bar{p}, \bar{q})=$ $0\}$. Because $\left(1 \otimes I_{2}\right)^{\top} \bar{q}=0$ and because the kernel of $L$ is spanned by 1 , the equality $\bar{q}^{\top} \mathcal{L} \bar{q}=0$ implies $\bar{q}=0$.

Let $(\bar{p}(t), \bar{q}(t))$ be a solution that belongs identically to $\mathrm{S}$. Then $\bar{q} \equiv 0$ and thus $\dot{\bar{q}} \equiv 0$ which means, according to the second equation of (15), that $\nabla V(\bar{p}) \equiv 0$. So the largest invariant set in $S$ is $M:=\{(\bar{p}, \bar{q}): \bar{q}=0$ and $\nabla V(\bar{p})=0\}$. Finally, $\bar{q}=0$ is equivalent to $\mathcal{L} q=0$ and $\nabla V(\bar{p})=0$ is equivalent to $\nabla V(p)=0$.

Remark 1: According to Theorem 3, if $\mathcal{G}$ is such that velocity consensus is achievable without shape control, and $\overline{\mathcal{G}}$ 
is such that formation shape control $\dot{p}=-\nabla V(p)$ for singleintegrator agents is achievable without velocity consensus, then both objectives are achievable via (12). Note that (12) combines the consensus control law with the shape control law, except that an additional integration is involved for the latter.

Remark 2: Obviously one could never hope to maintain a correct formation shape before velocity consensus has been achieved. Hence one can envisage that velocity consensus should be obtained on a faster time scale than that for shape control. The relative speeds of convergence can be crudely adjusted by including a positive multiplier of the matrix $\mathcal{L}$ in (12). Some simulations are given later in Section IV on how this gain can increase the convergence rate of the system.

We showed in the above theorem that all $(p, q)$ trajectories converge to the set $\Omega$ defined in (13) as $t$ goes to infinity. Note that when $\nabla V(p)=0$ the specified distance between the neighbor agents is not necessarily attained. (Consider, for example, the case where all agents are collocated, which is obviously not the desired shape. Then if $V(p)$ is in the form of (3), $\nabla V(p)$ is zero.)

We now define the set of desired formation shape and velocity in $(\bar{p}, \bar{q})$ frame, and investigate the conditions on the stability of this set. Let $\bar{H}$ be the incidence matrix associated with $\overline{\mathcal{G}}$ and $\overline{\mathcal{H}}=\bar{H} \otimes I_{2}$. If the graph $\overline{\mathcal{G}}$ has $r$ edges then $\overline{\mathcal{H}}$ is $2 n \times 2 r$. The vector of the aggregate relative positions of neighbor agents $e \in \mathbb{R}^{2 r}$ can now be defined as

$$
e=\left[\begin{array}{llll}
e_{1} & e_{2} & \cdots & e_{r}
\end{array}\right]^{\top}:=\overline{\mathcal{H}}^{\top} \bar{p} .
$$

Let

$$
\phi(e):=\left[\left\|e_{1}\right\|^{2}\left\|e_{2}\right\|^{2} \cdots\left\|e_{r}\right\|^{2}\right]^{\top}
$$

and

$$
g_{\bar{p}}(\bar{p}):=\phi\left(\overline{\mathcal{H}}^{\top} \bar{p}\right)=\phi(e)
$$

and let $\mathrm{d}^{*}$ be the vector the squares of the desired distances corresponding to $\phi(e)$. Then the set of correct equilibria for the desired formation shape and velocity can be represented by

$$
\Omega_{1}=\left\{(\bar{p}, \bar{q}): \bar{q}=0 \text { and } g_{\bar{p}}(\bar{p})=\mathrm{d}^{*}\right\} .
$$

We will show in the rest of this subsection, using the center manifold theorem, that if $V(p)$ is in the form of (3) then for the double-integrator model in (12), the inter-agent distance error represented by $\phi(e)-\mathrm{d}^{*}$ and the velocity error $\bar{q}$ of trajectories starting from a small neighborhood of the correct equilibrium in $\Omega_{1}$ converge to zero exponentially fast. Krick et. al. showed in [2] that for a shape control algorithm with a steepest descent control law, the set of correct equilibria representing the desired formation shape is locally asymptotically stable (Theorem 4 of [2]). We use the same idea to show that in the combined shape and velocity control, the trajectories starting from a sufficiently small neighborhood of the correct equilibrium set (representing the desired formation shape and velocity) converge to the set exponentially fast. We first make the following assumption:

Assumption 1: The framework $(\overline{\mathcal{G}}, p)$ is infinitesimally rigid at each $p$ where $\phi\left(\overline{\mathcal{H}}^{\top} p\right)=\mathrm{d}^{*}$.
According to the definition of infinitesimal rigidity, it is clear that if the framework $(\overline{\mathcal{G}}, p)$ is infinitesimally rigid, then the framework $(\overline{\mathcal{G}}, \bar{p})$ is also infinitesimally rigid.

To use the center manifold theorem, we first show that (15) can be written in the form of (8). Considering (18), the potential function (3) can be written as

$$
V(\bar{p})=\frac{1}{2}\left\|g_{\bar{p}}(\bar{p})-\mathrm{d}^{*}\right\|^{2}
$$

whose gradient $\nabla V(\bar{p})$ is

$$
\nabla V(\bar{p})=J_{g_{\bar{p}}}(\bar{p})^{\top}\left(g_{\bar{p}}(\bar{p})-\mathrm{d}^{*}\right) .
$$

By linearizing (15) at a point in $\Omega_{1}$ one has

$$
\left[\begin{array}{c}
\dot{\bar{p}} \\
\dot{\bar{q}}
\end{array}\right]=\left[\begin{array}{cc}
0 & I_{2 n} \\
-J_{g_{\bar{p}}}(\bar{p})^{\top} J_{g_{\bar{p}}}(\bar{p}) & -\mathcal{L}
\end{array}\right]\left[\begin{array}{c}
\bar{p} \\
\bar{q}
\end{array}\right] .
$$

Note that the matrix $J_{g_{\bar{p}}}(\bar{p})^{\top} J_{g_{\bar{p}}}(\bar{p})$ evaluated at a point in $\Omega_{1}$ has three zero eigenvalues and the rest are real and positive. This is because of the fact that the matrix $J_{g_{\bar{p}}} \in \mathcal{R}^{r \times 2 n}$ is the rigidity matrix associated with $\overline{\mathcal{G}}$ and has three zero eigenvalues if and only if the framework $(\overline{\mathcal{G}}, \bar{p})$ is infinitesimally rigid (see Theorem 2 of [2]). The matrix $\mathcal{L}=L \otimes I_{2}$ in (22) is also positive semi-definite and has two zero eigenvalues when the consensus graph $\mathcal{G}$ is connected.

To make the calculations easier, we perform a coordinate transformation that separates the centroid dynamics from the remaining dynamics of the system. Let $\mathcal{R} \in \mathbb{R}^{2 n \times 2 n}$ be an orthonormal matrix whose first two rows are $\frac{\mathbf{1}_{n}^{\top} \otimes I_{2}}{\sqrt{n}}$. Define

$$
\tilde{p}:=\mathcal{R} \bar{p}, \quad \tilde{q}:=\mathcal{R} \bar{q}, \quad \widetilde{\mathcal{H}}:=\mathcal{R} \overline{\mathcal{H}} .
$$

Then $\widetilde{\mathcal{H}}$ has the form $\widetilde{\mathcal{H}}=\left[0 \hat{\mathcal{H}}^{\top}\right]^{\top}$ where $\hat{\mathcal{H}}$ is a $(2 n-2) \times 2 r$ matrix which is because $\left(\mathbf{1}_{n} \otimes I_{2}\right)^{\top} \overline{\mathcal{H}}=0$. Let $\tilde{p}$ be partitioned as $\tilde{p}=\left[p^{o \top} \hat{p}^{\top}\right]^{\top}$ where $p^{o}$ is a 2 -vector and $\hat{p}$ is a $2 n-2$ vector. Then the vector of relative positions in (16) can be written as

$$
e=\overline{\mathcal{H}}^{\top} \bar{p}=\widetilde{\mathcal{H}}^{\top} \tilde{p}=\left[\begin{array}{ll}
0 & \hat{\mathcal{H}}^{\top}
\end{array}\right]\left[\begin{array}{c}
p^{o} \\
\hat{p}
\end{array}\right]=\hat{\mathcal{H}}^{\top} \hat{p} .
$$

Define

$$
g_{\tilde{p}}(\tilde{p}):=\phi\left(\tilde{\mathcal{H}}^{\top} \tilde{p}\right)=\phi(e)
$$

and consider (23). Then (22) can be written as

$$
\left[\begin{array}{c}
\dot{\tilde{p}} \\
\dot{\tilde{q}}
\end{array}\right]=\left[\begin{array}{cc}
0 & I_{2 n} \\
-J_{g_{\tilde{p}}}(\tilde{p})^{\top} J_{g_{\tilde{p}}}(\tilde{p}) & -\mathcal{R} \mathcal{L} \mathcal{R}^{\top}
\end{array}\right]\left[\begin{array}{c}
\tilde{p} \\
\tilde{q}
\end{array}\right]
$$

where $J_{g_{\tilde{p}}}(\tilde{p})=J_{g_{\bar{p}}}(\bar{p}) R^{\top}$. Since the first two rows of $\mathcal{R}$ are $\frac{\mathbf{1}_{n}^{\top} \otimes I_{2}}{\sqrt{n}}, \mathcal{L}=\overline{\mathcal{H}} \overline{\mathcal{H}}^{\top}$ and $\overline{\mathcal{H}}^{\top}\left(\mathbf{1}_{n} \otimes I_{2}\right)=0$, the first two rows and the first two columns of $\mathcal{R} \mathcal{L} \mathcal{R}^{\top}$ are zero and thus $\mathcal{R} \mathcal{L} \mathcal{R}^{\top}$ has the form

$$
-\mathcal{R} \mathcal{L R}^{\top} \tilde{q}=\left[\begin{array}{cc}
0_{2 \times 2} & 0 \\
0 & -\hat{\mathcal{L}}
\end{array}\right]\left[\begin{array}{c}
q^{o} \\
\hat{q}
\end{array}\right]
$$

where $q^{o}=\dot{p}^{o}, \hat{q}=\dot{\hat{p}}$, and the submatrix $\hat{\mathcal{L}}$ is positive definite. Let

$$
g_{\hat{p}}(\hat{p}):=\phi\left(\hat{\mathcal{H}}^{\top} \hat{p}\right)=\phi(e)
$$


Since $J_{g_{\tilde{p}}}(\tilde{p})=J_{g_{\bar{p}}}(\bar{p}) R^{\top}$ and $J_{g_{\bar{p}}}(\bar{p})\left(\mathbf{1}_{n} \otimes I_{2}\right)=0$ (see Lemma 1 and (21)), the first two rows and the first two columns of $J_{g_{\tilde{p}}}(\tilde{p})^{\top} J_{g_{\tilde{p}}}(\tilde{p})$ are also zero. Thus

$$
-J_{g_{\tilde{p}}}(\tilde{p})^{\top} J_{g_{\tilde{p}}}(\tilde{p}) \tilde{p}=\left[\begin{array}{cc}
0_{2 \times 2} & 0 \\
0 & -J_{g_{\hat{p}}}(\hat{p})^{\top} J_{g_{\hat{p}}}(\hat{p})
\end{array}\right]\left[\begin{array}{c}
p^{o} \\
\hat{p}
\end{array}\right]
$$

and the dynamics of the reduced order system can be written as

$$
\left[\begin{array}{c}
\dot{\hat{p}} \\
\dot{\hat{q}}
\end{array}\right]=\left[\begin{array}{cc}
0 & I_{2 n-2} \\
-J_{g_{\hat{p}}}(\hat{p})^{\top} J_{g_{\hat{p}}}(\hat{p}) & -\hat{\mathcal{L}}
\end{array}\right]\left[\begin{array}{c}
\hat{p} \\
\hat{q}
\end{array}\right] .
$$

Define the set

$$
\hat{\Omega}_{1}=\left\{(\hat{p}, \hat{q}): \hat{q}=0 \text { and } g_{\hat{p}}(\hat{p})=\mathrm{d}^{*}\right\}
$$

and note that this set is compact ${ }^{4}$ while the set $\Omega$ defined in (13) is not compact. Then because of (29) and the fact that $J_{g_{\bar{p}}}(\bar{p})^{\top} J_{g_{\bar{p}}}(\bar{p})$ has three zero eigenvalues at a point in $\Omega_{1}$ and the rest are real and positive, the matrix $J_{g_{\hat{p}}}(\hat{p})^{\top} J_{g_{\hat{p}}}(\hat{p})$ at a point in $\hat{\Omega}_{1}$ has one zero eigenvalue and the rest are real and positive.

Define now

$$
\hat{V}(\hat{p}):=\frac{1}{2}\left\|g_{\hat{p}}(\hat{p})-\mathrm{d}^{*}\right\|^{2}
$$

which according to (20) and $g_{\hat{p}}(\hat{p})=g_{\bar{p}}(\bar{p})=\phi(e)$ is equal to $V(\bar{p})$ in (20). Then the equations in (15) can be written, after straightforward calculations, as

$$
\left[\begin{array}{c}
\dot{\hat{p}} \\
\dot{\hat{q}}
\end{array}\right]=\left[\begin{array}{c}
\hat{q} \\
-\hat{\mathcal{L}} \hat{q}-\nabla \hat{V}(\hat{p})
\end{array}\right]:=F_{1}(\hat{p}, \hat{q})
$$

and the linearization of (33) at a point in $\hat{\Omega}_{1}$ can be represented by

$$
\begin{aligned}
{\left[\begin{array}{c}
\dot{\hat{p}} \\
\dot{\hat{q}}
\end{array}\right] } & =\left[\begin{array}{cc}
0 & I_{2 n-2} \\
-J_{g_{\hat{p}}}(\hat{p})^{\top} J_{g_{\hat{p}}(\hat{p})} & -\hat{\mathcal{L}}
\end{array}\right]\left[\begin{array}{c}
\hat{p} \\
\hat{q}
\end{array}\right] \\
& :=J_{F_{1}}(\hat{p}, \hat{q})\left[\begin{array}{c}
\hat{p} \\
\hat{q}
\end{array}\right] .
\end{aligned}
$$

As shown in Appendix 1, the matrix $J_{F_{1}}(\hat{p}, \hat{q})$ in (34) has one zero eigenvalue and the rest have negative real parts. So one can find a matrix $Q_{1}$ such that $Q_{1} J_{F_{1}}(\hat{p}, \hat{q}) Q_{1}^{-1}$ is in block diagonal form with a zero for the first term.

Define $\hat{z}$ as $\hat{z}:=\left[\begin{array}{ll}\hat{p}^{\top} & \hat{q}^{\top}\end{array}\right]^{\top}$. Then the $\hat{z}$ dynamics around a point $\hat{z}_{o}$ in $\hat{\Omega}_{1}$ can be written as

$$
\dot{\hat{z}}=J_{F_{1}}\left(\hat{z}_{o}\right) \hat{z}+\left(F_{1}(\hat{z})-J_{F_{1}}\left(\hat{z}_{o}\right) \hat{z}\right) .
$$

Define now $(\theta, \rho)=Q_{1} \hat{z}$. Then the $(\theta, \rho)$ dynamics have the form

$$
\begin{aligned}
\dot{\theta} & =h_{1}(\theta, \rho) \\
\dot{\rho} & =A_{s} \rho+h_{2}(\theta, \rho) .
\end{aligned}
$$

Without loss of generality, we assume $\hat{z}_{o}=0$. It can be easily verified that $h_{1}(\theta, \rho)$ and $h_{2}(\theta, \rho)$ in (36) satisfy $h_{1}(0,0)=0$,

\footnotetext{
${ }^{4}$ The compactness of $\hat{\Omega}_{1}$ can be shown using the facts that the set $\mathcal{C}=$ $\left\{(\hat{q}, e): \hat{q}=0\right.$ and $\left.\phi(e)=\mathrm{d}^{*}\right\}$ is a compact set and $\hat{\Omega}_{1}$ can be written as $\hat{\Omega}_{1}=\left\{(\hat{p}, \hat{q}): \hat{q}=0\right.$ and $\left.\hat{\mathcal{H}}^{\top} \hat{p}=e \in \mathcal{C}\right\}$. Since the kernel of $\hat{\mathcal{H}}^{\top}$ is zero, it follows that $\hat{\Omega}_{1}$ is also compact.
}

$h_{2}(0,0)=0, J_{h_{1}}(0,0)=0$ and $J_{h_{2}}(0,0)=0$. We showed up to here that (15) can be written in the form of (8).

Let the set $\hat{\mathcal{M}}$ be related to $\hat{\Omega}_{1}$ via the linear map $Q_{1}$ :

$$
\hat{\mathcal{M}}=\left\{(\theta, \rho):(\theta, \rho)=Q_{1} \hat{z} \text { and } \hat{z} \in \hat{\Omega}_{1}\right\} .
$$

The following lemma shows that $\hat{\mathcal{M}}$ is a center manifold for (36) with $(\theta, \rho)=Q_{1} \hat{z}$.

Lemma 2: The set $\hat{\mathcal{M}}$ in (37) is a center manifold for (36).

Proof: See Appendix 2.

We now state our main result in the following theorem:

Theorem 4: Suppose Assumption 1 holds and consider the double-integrator model in (12) and the transformations in (14). Let the potential function $V(p)$ in (12) be in the form of (3). Then there exists a neighborhood of the set $\Omega_{1}$ defined in (19) such that any trajectory starting in this neighborhood converges exponentially fast to a point in $\Omega_{1}$.

Proof: According to the center manifold theorem, we can conclude that there exists a function $h(\theta)$ such that in a neighborhood $\overline{\mathcal{N}}_{0}$ of $\hat{z}_{0}$ one has

$$
\hat{\mathcal{M}} \cap \overline{\mathcal{N}}_{0}=\{(\theta, \rho): \rho=h(\theta)\} .
$$

Since $\hat{\mathcal{M}}$ is an equilibrium manifold, $h_{1}(\theta, h(\theta))$ is zero on $\overline{\mathcal{N}}_{0}$ and the dynamics in (10) restricted to the center manifold $\hat{\mathcal{M}}$ is $\dot{\xi}=0$. Now by applying Theorem 2 the solutions of $(\theta, \rho)$ starting in $\overline{\mathcal{N}}_{0}$ are

$$
\begin{aligned}
& \theta(t)=\xi(t)+O\left(e^{-\gamma t}\right) \\
& \rho(t)=h(\xi(t))+O\left(e^{-\gamma t}\right) .
\end{aligned}
$$

So $(\theta(t), \rho(t))$ converges to $(\xi(0), h(\xi(0))) \in \hat{\mathcal{M}}$ exponentially fast which implies that $(\hat{p}(t), \hat{q}(t))$ converges to $Q_{1}^{-1}(\xi(0), h(\xi(0))) \in \hat{\Omega}_{1}$ exponentially fast. Since $\bar{p}(t)=$ $\mathcal{R}^{\top}\left[\begin{array}{ll}p^{o \top} & \hat{p}(t)^{\top}\end{array}\right]^{\top}$ and $\bar{q}(t)=\mathcal{R}^{\top}\left[\begin{array}{ll}q^{o \top} & \hat{q}(t)^{\top}\end{array}\right]^{\top}$ and both $p^{o}$ and $q^{o}$ are constant, then $\bar{p}(t)$ and $\bar{q}(t)$ converge to $\hat{\Omega}_{1}$ exponentially fast. Note that $\hat{\Omega}_{1}$ is compact and this argument can be repeated for any point in $\hat{\Omega}_{1}$ and can be lifted to $\Omega_{1}$ since $\sum_{n} \bar{q}_{i}(t)=0$ and $\sum_{n} \bar{p}_{i}(t)$ is constant.

Remark 3: Since the equilibrium manifold $\hat{\Omega}_{1}$ is compact and any trajectory starting from a small neighborhood of $\hat{\Omega}_{1}$ converges to $\hat{\Omega}_{1}$ exponentially fast, one can conclude that there exists a single exponent $\gamma$ such that all trajectories converge to $\hat{\Omega}_{1}$ from a small neighborhood at least as fast as $e^{-\gamma t}$. We call this manifold a locally exponentially stable manifold. (Note that for a non-compact manifold, although any trajectory starting from a sufficiently small neighborhood of the manifold converges to a point on the manifold exponentially fast, there might be no single $\gamma$ applicable to all trajectories.)

\section{B. Undirected consensus graph except for introduction of leader}

Suppose now that an arbitrary agent, without loss of generality agent 1 , is designated as a leader; equivalently, edges in the consensus graph $\mathcal{G}$ incident on that agent are all changed to be made outwardly directed while the remaining edges remain 
undirected. If before the change, the Laplacian matrix is (with $L_{11}$ a scalar)

$$
L=\left[\begin{array}{ll}
L_{11} & L_{12} \\
L_{21} & L_{22}
\end{array}\right]
$$

then after the change, the first row is replaced by 0 . Let the double-integrator model combining shape control and velocity consensus be written as

$$
\begin{aligned}
& \dot{p}=q \\
& \dot{q}=-\left(\left[\begin{array}{cc}
0 & 0 \\
L_{21} & L_{22}
\end{array}\right] \otimes I_{2}\right) q-\left[\begin{array}{cc}
0 & 0 \\
0 & I_{2 n-2}
\end{array}\right] \nabla V(p) .
\end{aligned}
$$

We shall assume that the formation is traveling between waypoints, and that the leader velocity is constant. Relaxing this assumption requires substantial adjustment. Let the leader velocity be denoted by $\alpha$. Let $v$ denote a vector whose $i$ th entry is $q_{i+1}-q_{1}$. Then it is easily verified that for the consensus problem without shape control, motion is governed by $\dot{v}=-\left(L_{22} \otimes I_{2}\right) v$; the constraints on $L$ assure that $L_{22}$ is positive definite, and so convergence is obvious. Suppose shape control is added to consensus. Let $z_{i}=p_{i+1}-p_{1}$ and observe that the translational invariance of $V(p)$ guarantees the existence of a function $\tilde{V}(z)$ with $V(p)=\tilde{V}(z)$. Then equations (41) are evidently replaced by

$$
\begin{aligned}
\dot{z} & =v \\
\dot{v} & =-\left(L_{22} \otimes I_{2}\right) v-\nabla \tilde{V}(z) .
\end{aligned}
$$

Note that the motion of the leader agent is neither affected by the velocity of other agents nor any errors in distances between it and its adjacent agents in $\overline{\mathcal{G}}$. We have the following result.

Theorem 5: Let the double-integrator model of the agents be given by (41). Suppose agent 1 is the leader of the formation and is traveling between waypoints, and the leader velocity is constant between the waypoints and is denoted by $\alpha$. Suppose the edges in $\mathcal{G}$ incident on agent 1 are all outwardly directed while the remaining edges in $\mathcal{G}$ are undirected, and $\mathcal{G}$ is rooted at the leader. Assume the formation shape graph $\overline{\mathcal{G}}$ is rigid. Then all trajectories tend as $t \rightarrow \infty$ to the set

$$
\Omega_{2}=\{(p, q): q=\alpha \mathbf{1} \text { and } \nabla V(p)=0\} .
$$

Proof: We start with (42) and consider the Lyapunov function $\tilde{W}(z, v)=(1 / 2) v^{\top} v+\tilde{V}(z)$, for which $\dot{\tilde{W}}=$ $-v^{\top}\left(L_{22} \otimes I_{2}\right) v$. It is immediate that $v$ is bounded, as is $\tilde{V}(z)=V(p)$. Boundedness of $\tilde{V}(z)=V(p)$ ensures that all edge lengths in the formation graph $\overline{\mathcal{G}}$ are bounded, and so all $z_{i}$ are bounded. The LaSalle's Invariance Principle then easily shows that all trajectories converge to the set $\{(z, v): \nabla \tilde{V}(z)=0, v=0\}$. The condition $v=0$ corresponds to consensus with leader velocity $\alpha$ and the condition $\nabla \tilde{V}(z)=0$ is equivalent to $\nabla V(p)=0$. To see that this implies that $\nabla V(p)=0$, observe using the definition of the $z_{i}$ and Lemma 1 that

$$
\begin{aligned}
\frac{\partial}{\partial z_{1}} \tilde{V}(z) & =\frac{\partial}{\partial p_{2}} V(p)-\frac{\partial}{\partial p_{1}} V(p) \\
\frac{\partial}{\partial z_{2}} \tilde{V}(z) & =\frac{\partial}{\partial p_{3}} V(p)-\frac{\partial}{\partial p_{1}} V(p) \\
& \vdots \\
\frac{\partial}{\partial z_{n-1}} \tilde{V}(z) & =\frac{\partial}{\partial p_{n}} V(p)-\frac{\partial}{\partial p_{1}} V(p) \\
0 & =\frac{\partial}{\partial p_{1}} V(p)+\cdots+\frac{\partial}{\partial p_{n}} V(p)
\end{aligned}
$$

and it is clear that $\nabla \tilde{V}(z)=0$ iff $\nabla V(p)=0$.

Similarly to the case where the consensus graph is undirected and there is no leader in the formation, it can be shown using the center manifold theorem that the set of correct equilibria is locally exponentially stable. Define the matrix $T \in \mathbb{R}^{(2 n-2) \times 2 n}$ as $z=T p$ (or equivalently $v=T q$ ) where $z \in \mathbb{R}^{2 n-2}$ and $v \in \mathbb{R}^{2 n-2}$ are respectively the vectors of all $z_{i}$ and $v_{i}$ stacked together, and let

$$
g_{z}(z):=\phi(e) .
$$

Similarly to (20), the potential function $\tilde{V}(z)$ in (42) can be written as

$$
\tilde{V}(z)=\frac{1}{2}\left\|g_{z}(z)-\mathrm{d}^{*}\right\|^{2}
$$

whose gradient $\nabla \tilde{V}(z)$ is

$$
\nabla \tilde{V}(z)=J_{g_{z}}(z)^{\top}\left(g_{z}(z)-\mathrm{d}^{*}\right) .
$$

The set of correct equilibrium points can also be written as

$$
\hat{\Omega}_{2}=\left\{(z, v): v_{i}=0 \forall i \text {, and } g_{z}(z)=\mathrm{d}^{*}\right\} .
$$

Note that $\hat{\Omega}_{2}$ is a compact set. Consider (42) and define $F_{2}(z, v)$ as

$$
\left[\begin{array}{c}
\dot{z} \\
\dot{v}
\end{array}\right]=\left[\begin{array}{c}
v \\
-\left(L_{22} \otimes I_{2}\right) v-\nabla \tilde{V}(z)
\end{array}\right]:=F_{2}(v, z) .
$$

Then by linearizing (48) at a point in $\hat{\Omega}_{2}$ one has

$$
\begin{aligned}
{\left[\begin{array}{c}
\dot{z} \\
\dot{v}
\end{array}\right] } & =\left[\begin{array}{cc}
0 & I_{2 n-2} \\
-J_{g_{z}}(z)^{\top} J_{g_{z}}(z) & -L_{22} \otimes I_{2}
\end{array}\right]\left[\begin{array}{c}
z \\
v
\end{array}\right] \\
& :=J_{F_{2}}(z, v)\left[\begin{array}{c}
z \\
v
\end{array}\right]
\end{aligned}
$$

where $J_{g_{p}}(p)=J_{g_{z}}(z) T$. From the definition of $T$, it is easy to see that the rank of $T$ is $2 n-2$. According to Sylvester's rank inequality and the fact that $\operatorname{rank}\left(J_{g_{p}}(p)\right) \leq$ $\min \left\{\operatorname{rank}\left(J_{g_{z}}(z)\right), \operatorname{rank}(T)\right\}$, then $J_{g_{p}}(p)$ has the same rank as $J_{g_{z}}(z)$. Since the rank of $J_{g_{p}}(p)=J_{g_{z}}(z) T$ is $2 n-3$, because it is the rank of the rigidity matrix associated with the graph $\overline{\mathcal{G}}$ and the framework $(\overline{\mathcal{G}}, p)$ is assumed to be infinitesimally rigid, the rank of $J_{g_{z}}(z)$ is also $2 n-3$. Since $J_{g_{z}}(z)$ is $r \times(2 n-2)$, the nullity of $J_{g_{z}}(z)$ and also the nullity of $J_{g_{z}}(z)^{\top} J_{g_{z}}(z)$ is 1 . So $J_{g}(z)^{\top} J_{g}(z)$ has one zero eigenvalue. According to the structure of $J_{g}(z)^{\top} J_{g}(z)$, it is clear that $J_{g}(z)^{\top} J_{g}(z)$ is symmetric and positive semidefinite. Thus the nonzero eigenvalues are all real and positive.

Since $L_{22}$ is a symmetric positive definite matrix, it can be shown, similarly to Lemma 5 , that $J_{F_{2}}(z, v)$ in (49) has only 
one zero eigenvalue and the rest have negative real parts. Then we can conclude that any trajectory of $(z(t), v(t))$ starting from a small neighborhood of $\hat{\Omega}_{2}$ in (47) converges to a point in $\hat{\Omega}_{2}$ exponentially fast.

\section{Directed consensus graph}

In this subsection, we shall suppose that the initially given consensus graph $\mathcal{G}$ is directed and meets the conditions of Theorem 1. We proceed straight to the situation where a leader is selected. The following lemma is pertinent.

Lemma 3: Let $\mathcal{G}$ be a weakly connected directed $\operatorname{graph}^{5}$ with a unique closed strongly connected component. Choose any vertex, call it $\nu$, belonging to the closed strongly connected component as a leader, and remove the edges in $\mathcal{G}$ directed towards $\nu$. Call $\mathcal{G}^{\prime}$ the new graph. Then $\mathcal{G}^{\prime}$ has a closed strongly connected component which is unique, being in fact the single vertex $\nu$, and accordingly consensus with convergence will occur.

Proof: Let $\mathcal{G}^{*}$ be the closed strongly connected component of $\mathcal{G}$, and let $\mathcal{V}^{*}$ denote its vertex set. If $\left|\mathcal{V}^{*}\right|=1$, then $\mathcal{G}^{\prime}=\mathcal{G}$ and the result is trivial. So suppose $\left|\mathcal{V}^{*}\right|>1$. Since $\mathcal{G}^{*}$ is strongly connected, from any selected vertex in $\mathcal{G}^{*}$, there exists a path to any other vertex in $\mathcal{G}^{*}$. In particular, there exists a spanning tree $\mathcal{T}^{*}$ in $\mathcal{G}^{*}$ for which $\nu$ is a root vertex, i.e. has no incoming edges. Also, the hypothesis of the lemma guarantees there is a spanning tree for all of $\mathcal{G}$. All edges in this spanning tree must be outgoing from $\mathcal{G}^{*}$ because it is closed. It is clear that the edges of this spanning tree lying in $\mathcal{G}^{*}$ could be replaced by those of $\mathcal{T}^{*}$ and one would still have a spanning tree of $\mathcal{G}$. In this new spanning tree of $\mathcal{G}, \nu$ is the root vertex, and so this spanning tree of $\mathcal{G}$ is also a spanning tree of $\mathcal{G}^{\prime}$. Since $\mathcal{G}^{\prime}$ has a spanning tree, it has a closed strongly connected component which is unique (and the component defined by $\nu$ alone is closed and strongly connected), and the lemma is proved.

With $\mathcal{G}^{\prime}$ as defined in the above lemma, let $\nu$ be the identified vertex, and suppose it corresponds to agent 1 . Let $L^{\prime}$ be the associated Laplacian matrix. Then $L^{\prime}$ has the following form:

$$
L^{\prime}=\left[\begin{array}{cc}
0 & 0 \\
L_{21}^{\prime} & L_{22}^{\prime}
\end{array}\right]
$$

Observe that since the consensus property is guaranteed, $L^{\prime}$ has precisely one zero eigenvalue and the other eigenvalues have positive real part. Hence all eigenvalues of $L_{22}^{\prime}$ have positive real part. This means, see [3], that there exists a diagonal positive definite matrix $P$ such that

$$
Q:=P L_{22}^{\prime}+L_{22}^{\prime \top} P>0 \text {. }
$$

We now have the following result.

Theorem 6: Suppose there is a directed consensus graph $\mathcal{G}^{\prime}$ which is rooted at the leader with the leader defined by agent 1 , and an underlying formation shape graph $\overline{\mathcal{G}}$ which is rigid. Let $\mathcal{L}^{\prime}=L^{\prime} \otimes I_{2}$ with $L^{\prime}$ the Laplacian associated with $\mathcal{G}^{\prime}$, and let $V(p)$ be a function invariant to translation,

\footnotetext{
${ }^{5} \mathrm{~A}$ directed graph is weakly connected if there is an undirected path between each pair of distinct vertices [18].
}

rotation or reflection serving as the basis of a steepest descent law, suppose the shape control law is $\dot{p}=-\nabla V(p)$. For a diagonal positive definite $P$ satisfying (51), consider the double-integrator model combining shape control and velocity consensus:

$$
\begin{aligned}
\dot{p} & =q \\
\dot{q} & =-\mathcal{L}^{\prime} q-\left[\begin{array}{cc}
0_{2 \times 2} & 0 \\
0 & P^{-1} \otimes I_{2}
\end{array}\right] \nabla V(p)
\end{aligned}
$$

which includes the equation $\dot{q}_{1}=0$, (and ensures distributed control action for shape adjustment). Then all trajectories tend as $t \rightarrow \infty$ to the set

$$
\Omega_{3}=\left\{(p, q): q_{i}=q_{1} \forall i \text {, and } \nabla V(p)=0\right\} .
$$

Proof: First transform the equations by setting $v_{i}=$ $q_{i+1}-q_{1}, z_{i}=p_{i+1}-p_{1}$. Let $\tilde{V}(z)$ be such that $V(p)=\tilde{V}(z)$, existence being guaranteed by the hypotheses on $V(p)$. The adjusted equations are

$$
\begin{aligned}
\dot{z} & =v \\
\dot{v} & =-\left(L_{22}^{\prime} \otimes I_{2}\right) v-\left(P^{-1} \otimes I_{2}\right) \nabla \tilde{V}(z) .
\end{aligned}
$$

Adopt as a Lyapunov function

$$
W(z, v)=\frac{1}{2} v^{\top}\left(P \otimes I_{2}\right) v+\tilde{V}(z) .
$$

Its time derivative along trajectories is $\dot{W}=-v^{\top}\left(Q \otimes I_{2}\right) v$. Boundedness of $v, z$ follows as earlier for (42), and so by the LaSalle's Invariance Principle, the consensus property is immediate. A further minor step establishes $\nabla \tilde{V}(z)=0$ which is equivalent to $\nabla V(p)=0$. Hence the theorem is proved.

The set of correct equilibria can be defined by

$$
\hat{\Omega}_{3}=\left\{(z, v): v_{i}=0 \forall i \text {, and } g_{z}(z)=\mathrm{d}^{*}\right\} .
$$

Similarly to the previous subsection, it can be shown using the center manifold theorem that the trajectories starting from a small neighborhood of $\hat{\Omega}_{3}$ in (53) converge to $\hat{\Omega}_{3}$ exponentially fast. Consider (52) and define $F_{3}(z, v)$ as

$$
\left[\begin{array}{c}
\dot{z} \\
\dot{v}
\end{array}\right]=\left[\begin{array}{c}
v \\
-\left(L_{22} \otimes I_{2}\right) v-\left(P^{-1} \otimes I_{2}\right) \nabla \tilde{V}(z)
\end{array}\right]:=F_{3}(z, v) .
$$

Then by linearizing (57) at a point in (56) one has

$$
\begin{aligned}
{\left[\begin{array}{c}
\dot{z} \\
\dot{v}
\end{array}\right] } & =J_{F_{3}}(z, v)\left[\begin{array}{c}
z \\
v
\end{array}\right] \\
& =\left[\begin{array}{cc}
0 & I_{2 n-2} \\
-\left(P^{-1} \otimes I_{2}\right) J_{g_{z}}(z)^{\top} J_{g_{z}}(z) & -L_{22} \otimes I_{2}
\end{array}\right]\left[\begin{array}{c}
z \\
v
\end{array}\right] .
\end{aligned}
$$

As explained in the previous subsection, the matrix $-J_{g}(z)^{\top} J_{g}(z)$ is symmetric and negative semidefinite which has one zero eigenvalue and the nonzero eigenvalues are all real and negative. As the matrix $P$ in (58) is positive definite, then according to Theorem 7.6.3 of [17], $-\left(P^{-1} \otimes\right.$ $\left.I_{2}\right) J_{g}(z)^{\top} J_{g}(z)$ has the same number of zero, positive, and negative eigenvalues as $-J_{g}(z)^{\top} J_{g}(z)$, that is $-\left(P^{-1} \otimes\right.$ $\left.I_{2}\right) J_{g}(z)^{\top} J_{g}(z)$ has one zero eigenvalue and the rest are real and negative.

Now we want to show that $J_{F_{3}}(z, v)$ in (58) has only one zero 
eigenvalue, i.e, the dimension of the kernel of this matrix is one. let $\left[x^{\top} y^{\top}\right]^{\top} \in \mathbb{R}^{4 n-4}$ be the eigenvector associated with the zero eigenvalue of $J_{F_{3}}(z, v)$. Thus

$$
\left[\begin{array}{cc}
0 & I_{2 n-2} \\
-\left(P^{-1} \otimes I_{2}\right) J_{g_{z}}(z)^{\top} J_{g_{z}}(z) & -L_{22}^{\prime} \otimes I_{2}
\end{array}\right]\left[\begin{array}{l}
x \\
y
\end{array}\right]=0
$$

Then $y=0$ and $-\left(P^{-1} \otimes I_{2}\right) J_{g_{z}}(z)^{\top} J_{g_{z}}(z) x=0$. Since there exists only one independent vector $x$ that spans the null space of $-\left(P^{-1} \otimes I_{2}\right) J_{g_{z}}(z)^{\top} J_{g_{z}}(z)$, one can conclude that there exists only one independent vector $\left[x^{\top} y^{\top}\right]^{\top}=\left[\begin{array}{ll}x^{\top} & 0\end{array}\right]^{\top}$ that spans the null space of $J_{F_{3}}(z, v)$. Then it can be shown that $J_{F_{3}}(z, v)$ has only one zero eigenvalue. The rest of the local exponential convergence proof is similar to the proofs in the previous subsections.

\section{Directed consensus graph with a closed strongly connected subgraph}

In this subsection, we suppose the consensus graph $\mathcal{G}$ has a unique closed strongly connected component, which contains more than a single vertex, while $\mathcal{G}$ itself is not strongly connected. We first investigate the consensus problem and provide a Lyapunov function for the stability proof and then go to the consensus and shape control problem.

1) Velocity consensus:

Suppose the matrix $L \in \mathbb{R}^{n \times n}$ is partitioned as

$$
L=\left[\begin{array}{ll}
A & 0 \\
B & C
\end{array}\right]
$$

where $A \in \mathbb{R}^{m \times m}$ is square and is the Laplacian of the subgraph of $\mathcal{G}$ comprising the closed strongly connected component which contains $m$ vertices. Hence, as noted in Theorem 1 , there is a positive vector $\eta_{a}$ spanning the cokernel of $A$, and $\eta=\left[\begin{array}{ll}\eta_{a}^{\top} & 0\end{array}\right]^{\top}$ spans the cokernel of $L$.

Define $\mathcal{A}:=A \otimes I_{2}, \mathcal{B}:=B \otimes I_{2}, \mathcal{C}:=C \otimes I_{2}$, and $\mathcal{L}$ by

$$
\mathcal{L}=L \otimes I_{2}=\left[\begin{array}{ll}
\mathcal{A} & 0 \\
\mathcal{B} & \mathcal{C}
\end{array}\right]
$$

We shall prove the stability of the velocity consensus problem with the aid of the following lemma.

Lemma 4: With notation as above, there exists a nonsingular positive definite $\mathcal{P}$ and a nonnegative definite $\mathcal{Q}$ with kernel spanned by $\left(\mathbf{1} \otimes I_{2}\right)$ such that

$$
\mathcal{P} \mathcal{L}+\mathcal{L}^{\top} \mathcal{P}=\mathcal{Q} .
$$

Proof: Recall that $\left(\eta_{a} \otimes I_{2}\right)^{\top} \mathcal{A}=0$ and $\mathcal{L}\left(\mathbf{1} \otimes I_{2}\right)=0$. Because the entries of $\eta_{a}$ are positive, the inner product $\eta_{a}^{\top} \mathbf{1}$ is nonzero. It is trivial that the matrix

$$
I_{2 n}-\left(\mathbf{1} \otimes I_{2}\right) \frac{\left[\left(\eta_{a} \otimes I_{2}\right)^{\top} 0_{2 \times 2(n-m)}\right]}{\eta_{a}^{\top} \mathbf{1}}
$$

has rank $2 n-2$, and so has a factorisation as

$$
I_{2 n}-\left(\mathbf{1} \otimes I_{2}\right) \frac{\left[\left(\eta_{a} \otimes I_{2}\right)^{\top} 0\right]}{\eta_{a}^{\top} \mathbf{1}}=M N
$$

where $M, N$ are $2 n \times(2 n-2)$ and $(2 n-2) \times 2 n$ respectively, both with rank $2 n-2$. Define the $2 n \times 2 n$ matrix

$$
U=\left[\left(\mathbf{1} \otimes I_{2}\right) M\right]
$$

and it is easily verified that with

$$
S=\left[\begin{array}{c}
\frac{\left[\left(\eta_{a} \otimes I_{2}\right)^{\top} 0\right]}{\eta_{a}^{\top} \mathbf{1}} \\
N
\end{array}\right]
$$

there holds $U S=I$, i.e. $S=U^{-1}$. Then $S U=I_{2 n}$ and the entries of $S U$ give $N\left(\mathbf{1} \otimes I_{2}\right)=0$, a fact used below. A short calculation also shows that

$$
U^{-1} \mathcal{L} U=\left[\begin{array}{cc}
0_{2 \times 2} & 0_{2 \times(2 n-2)} \\
0_{(2 n-2) \times 2} & N \mathcal{L} M
\end{array}\right] .
$$

Because the matrix $L$ has one zero eigenvalue and the remainder with positive real parts, the $\mathcal{L}$ has two zero eigenvalues and the remainder with positive real parts. Hence all eigenvalues of $N \mathcal{L} M$ have positive real parts. So there exist positive definite symmetric $\hat{P}, \hat{Q}$ such that

$$
\hat{P}(N \mathcal{L} M)+(N \mathcal{L} M)^{\top} \hat{P}=\hat{Q} .
$$

Then equation (62) is satisfied with the definitions

$\mathcal{P}=U^{-\top}\left[\begin{array}{cc}I_{2} & 0 \\ 0 & \hat{P}\end{array}\right] U^{-1}, \quad \mathcal{Q}=U^{-\top}\left[\begin{array}{cc}0_{2 \times 2} & 0 \\ 0 & \hat{Q}\end{array}\right] U^{-1}$.

It is evident that the function $q^{\top} \mathcal{P} q$ is a Lyapunov function establishing stability of the consensus system. By Lasalle, the solutions approach a limit point in the set $\mathcal{Q} q=0$. Equivalently, the limiting solutions must obey

$$
U^{-\top}\left[\begin{array}{cc}
0_{2 \times 2} & 0 \\
0 & \hat{Q}
\end{array}\right]\left[\begin{array}{c}
\frac{\left[\left(\eta_{a} \otimes I_{2}\right)^{\top} 0\right]}{\eta_{a}^{\top} 1} \\
N
\end{array}\right] q=0 .
$$

It is trivial that $N q=0$. As noted above, the nullspace of $N$ is spanned by $1 \otimes I_{2}$. So

$$
q=\left(\mathbf{1} \otimes I_{2}\right) \mu
$$

for some $\mu \in \mathbb{R}^{2}$.

2) Combined velocity and shape control:

The equations of motion we adopt are:

$$
\begin{aligned}
\dot{p} & =q \\
\dot{q} & =-\mathcal{L} q-\mathcal{P}^{-1} \nabla V(p) .
\end{aligned}
$$

It should be noted that $\mathcal{P}$ is not a diagonal matrix and thus information from other than neighbour agents might be needed to form the acceleration of a particular agent. In other words, when the consensus graph has a unique closed strongly connected component which contains more than a single vertex, the control law might not be completely distributed.

Now choose as a 'Lyapunov-like' function

$$
W=\frac{1}{2} q^{\top} \mathcal{P} q+V(p) .
$$

It is straightforward to check that this results in

$$
\dot{W}=-\frac{1}{2} q^{\top} \mathcal{Q} q \text {. }
$$

It is immediate that $q$ is bounded. However, it is not immediate that $p$ is bounded. Nevertheless, $V(p)$ is bounded, from which it easily follows that every $\left\|p_{i}-p_{j}\right\|$ is bounded, 
Consequently, $\dot{q}$ is bounded, and so $\ddot{W}$ is bounded. Under these circumstances, Barbalat's lemma implies that $\dot{W} \rightarrow 0$. The calculation for the consensus only problem then shows that

$$
q \rightarrow\left(\mathbf{1} \otimes I_{2}\right) \mu
$$

for some 2-vector $\mu$. At this point, it has not been established that $\mu$ is constant. Insert this into (67). The conclusion is for the equilibrium motions

$$
\begin{aligned}
\dot{p} & =\left(\mathbf{1} \otimes I_{2}\right) \mu \\
\left(\mathbf{1} \otimes I_{2}\right) \dot{\mu} & =-\mathcal{P}^{-1} \nabla V(p) .
\end{aligned}
$$

Multiply the second equation on the left by $\left(1 \otimes I_{2}\right)^{\top} \mathcal{P}$. The right side is zero since $\left(\mathbf{1} \otimes I_{2}\right)^{\top} \nabla V(p)=0$, and because $\mathbf{1}^{\top} \mathcal{P} \mathbf{1}>0$, there results $\dot{\mu}=0$, so that $\mu$ is constant. The second equation of (67) then has $\dot{q}=0, \mathcal{L} q=0$ (recall that $1 \otimes I_{2}$ is a null vector of $\mathcal{L}$ ), and so $\nabla V(p)=0$.

This establishes (for a rigid formation) that simultaneous velocity consensus and local shape control have been achieved.

The proof for local exponential stability of the desired equilibrium set is similar to the previous subsections and is omitted for brevity. Similarly to the previous subsections, the equilibrium set is not compact in this case. So one should first reduce the order of the system and find a compact equilibrium manifold for the reduced order system and then show that the equilibrium manifold is locally exponentially stable. Alternatively, one can start with a non-compact equilibrium set and use the generalization of the center manifold theorem in [9] to show that the trajectories starting from a compact neighborhood of the non-compact equilibrium manifold converge to a compact subset of the equilibrium manifold exponentially fast.

\section{Simulations}

In this section, we simulate the results in Section III for a 5-agent formation. In all simulations in this section, we assume the initial position and velocity of the agents are: $p_{1}(0)=[-3.5,0]^{\top}, p_{2}(0)=[-1.75,0]^{\top}, p_{3}(0)=[0,0]^{\top}$, $p_{4}(0)=[1.75,0]^{\top}, p_{5}(0)=[3.5,0]^{\top}, q_{1}(0)=[-0.5,1]^{\top}$, $q_{2}(0)=[1,3]^{\top}, q_{3}(0)=[3,0]^{\top}, q_{4}(0)=[1,-1]^{\top}$, and $q_{5}(0)=[0,1]^{\top}$. We start with a formation whose consensus graph is undirected. Let the topology of the consensus graph $\mathcal{G}$ and the topology of the shape control graph $\overline{\mathcal{G}}$ be as shown in Fig. 2(a) and Fig. 2(b), respectively. Note that $\mathcal{G}$ is connected and $\overline{\mathcal{G}}$ is rigid. The desired distances between the neighbor agents in $\overline{\mathcal{G}}$ are assumed to be $d_{12}^{*}=d_{15}^{*}=\sqrt{2}, d_{23}^{*}=d_{34}^{*}=$ $d_{45}^{*}=d_{52}^{*}=2, d_{35}^{*}=d_{24}^{*}=\sqrt{8}$ and $d_{14}^{*}=\sqrt{10}$. We assume that $V(p)$ in (12) is in the form of (3).

The agents' trajectories when the dynamics of the system are as (12) are shown Fig. 3. As explained earlier, the algorithms proposed in this paper control the formation shape and the velocity of agents, but they do not control the orientation and the position of the center of mass of the formation.

As explained in Remark 2, the rate of convergence of the consensus term can affect the convergence time of the formation to the desired shape and velocity. We consider two different cases where the consensus term in (12) is multiplied

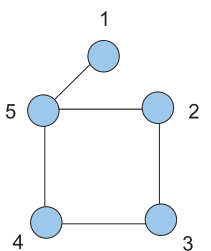

(a) $\mathcal{G}$

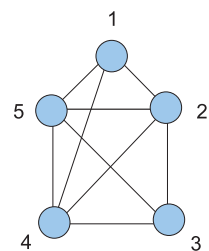

(b) $\overline{\mathcal{G}}$
Fig. 2: Consensus graph $\mathcal{G}$ and shape control graph $\overline{\mathcal{G}}$ used in the simulation of the double-integrator model in (12).

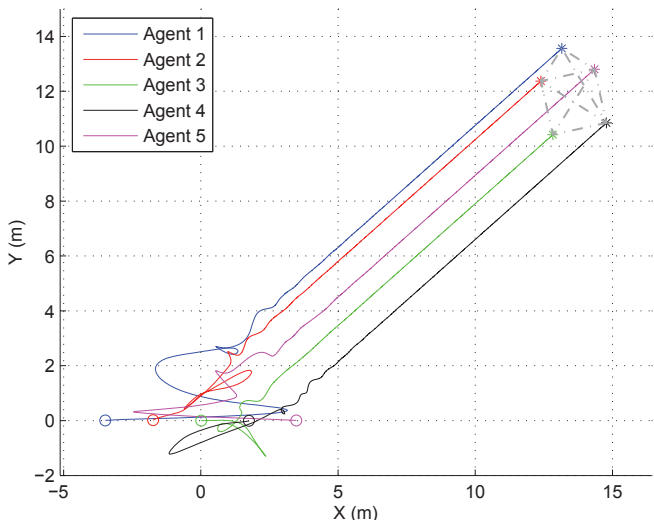

Fig. 3: Simulation results when the consensus graph is undirected and the system dynamics are governed by (12) with $V(p)$ given in (3). The position of agents at $t=0$ and $t=15 \mathrm{sec}$ are respectively shown by ' $\circ$ ' and '*'. The dasheddotted lines represent the formation links associated with the edges in $\overline{\mathcal{G}}$.

by $k=1$ and $k=10$. The trajectories of the agents when $k=10$ are shown in Fig. 4, and the sum of the distance error of the neighbor agents and the sum of the 2-norm of velocity error between the agents for $k=1$ and $k=10$ are shown in Fig. 5. It can be seen in Fig. 5 that the convergence rate of the system increases as $k$ changes from 1 to $10 .^{6}$

To check the robustness of the system against noise, we assume that the velocity measurements used for consensus are contaminated by Gaussian noise. Note that robustness against noise is to be expected because of the exponential convergence. We consider the following double-integrator model of the system in this case

$$
\begin{aligned}
\dot{p} & =q \\
\dot{q} & =-k(\mathcal{L} q+\nu)-\nabla V(p) .
\end{aligned}
$$

where $k$ is the consensus gain which is assumed to be 10 , and $\nu$ is the vector of all velocity measurement noises $\nu_{i}$ at the agents $i=1 \cdots, 5$ stacked together. We assume $\nu_{i}$ is zero mean Gaussian noise with variance 0.25 . Simulation results comparing the noiseless case with the noisy case are shown in Fig. 6.

${ }^{6}$ Further simulations show that if the consensus gain increases too much,
the convergence of the inter-agent distances to the desired distances becomes slower. 


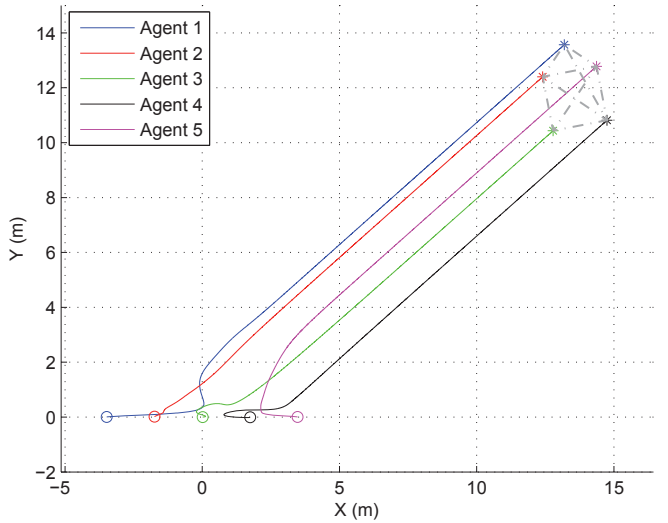

Fig. 4: Simulation results when the consensus graph is undirected and the system dynamics are governed by (12) with $V(p)$ given in (3). We assume the consensus term in (12) is multiplied by a gain $k=10$. The position of agents at $t=0$ and $t=15 \mathrm{sec}$ are respectively shown by ' $\circ$ ' and ' $*$ '. The dashed-dotted lines represent the formation links associated with the edges in $\overline{\mathcal{G}}$.
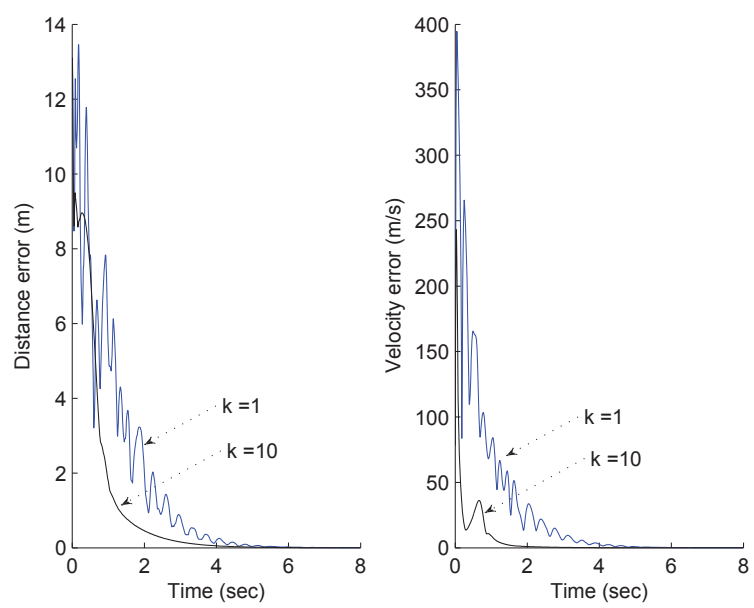

Fig. 5: The sum of the distance errors between the neighbor agents and the sum of the 2-norm of the vector of velocity errors when the system dynamics are governed by (12) (blue line) and when the system dynamics are governed by (12) but the consesnsus term is multplied by a gain $k=10$ (black line).

Now suppose the consensus graph has a leader which is denoted as agent 1 . We first assume that the consensus graph $\mathcal{G}$ is still undirected except that the edges in $\mathcal{G}$ incident on agent 1 are all changed to be made outwardly directed. We assume that the speed of the leader changes at some waypoints (which are sufficiently far from each other) and remains constant between the waypoints. We continue to assume that the consensus term in (41) is multiplied by $k=10$ and $V(p)$ is as (3). The trajectories of the agents are shown in Fig. 7. The initial positions and velocities of the agents in Fig. 7 are the same as Fig. 3.

Next assume that the consensus graph is directed as shown in Fig. 8. We now compare the distance and velocity error
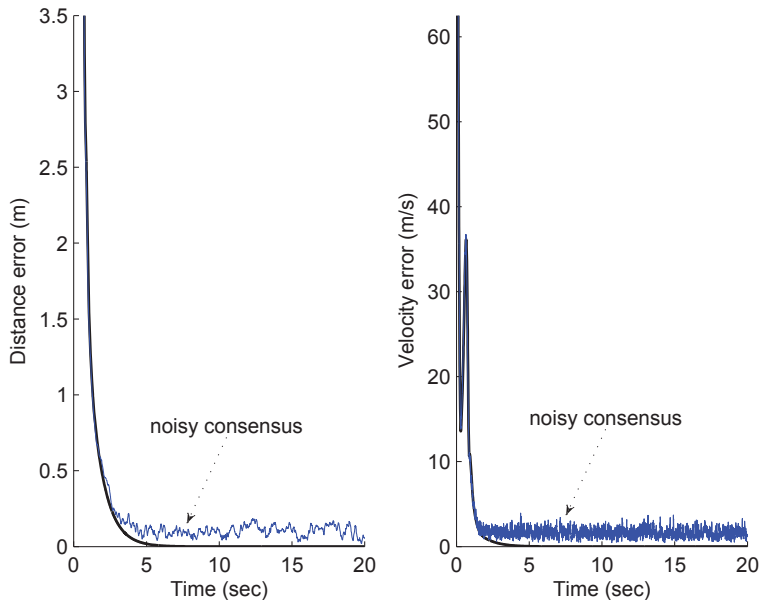

Fig. 6: The sum of the distance errors between the neighbor agents and the sum of the 2-norm of the vector of velocity errors when the system dynamics are governed by (12), the consensus term is multiplied by $k=10$, and the velocity measuerements are noiseless (black) ans noisy (blue).

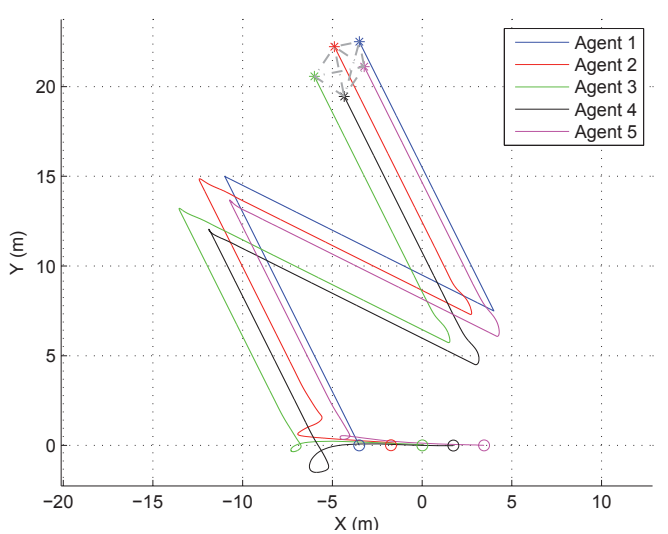

Fig. 7: Simulation results when the consensus graph is undirected except that the edges incident on agent 1 are all outwardly directed, and when the speed of the leader changes at two waypoints. The position of agents at $t=0$ and $t=15 \mathrm{sec}$ are respectively shown by 'o' and ' $*$ '.

of three cases where the the models of the system are as (12), (42), and (52). We assume that the consensus term is multiplied by a gain $k=10$ and $V(p)$ is as (3). The sum of the distance errors between the neighbor agents and the sum of the 2-norm of the vector of velocity errors for these cases are shown in Fig. 9. It can be seen that all errors converge to zero, and when the agents in the formation have more knowledge about their neighbors, the errors converge to zero faster.

We finally assume that the consensus graph is not strongly connected, but has a closed strongly connected component. The consensus graph we consider here is shown in Fig. 10. The matrix $\mathcal{P}$ in (67) can be found using Lemma 4. A possible 


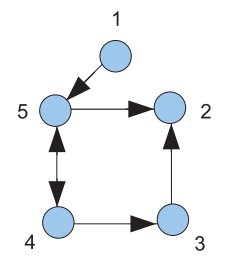

Fig. 8: Consensus graph $\mathcal{G}$ used in the simulation of (52).
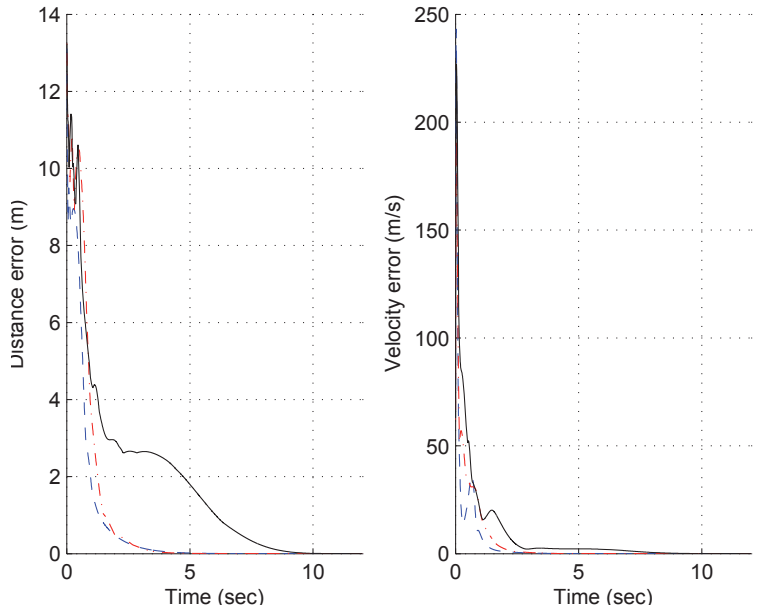

Fig. 9: The sum of the distance errors between the neighbor agents and the 2-norm of the vector of velocity errors for the cases where the consensus graph is undirected (dashed blue line), is undirected except that the edges from the leader are outwardly directed (dashed-dotted red line) and is directed (solid black line).

$$
\begin{aligned}
& \mathcal{P} \text { is } \\
& \mathcal{P}=\left[\begin{array}{rrrrr}
1.224 & 0.224 & -0.316 & -0.316 & -0.316 \\
0.224 & 1.224 & -0.316 & -0.316 & -0.316 \\
-0.316 & -0.316 & 0.877 & -0.122 & -0.122 \\
-0.316 & -0.316 & -0.122 & 0.877 & -0.122 \\
-0.316 & -0.316 & -0.122 & -0.122 & 0.877
\end{array}\right]_{(72)}
\end{aligned}
$$

The distance and velocity errors in this case are shown in Fig. 11 .

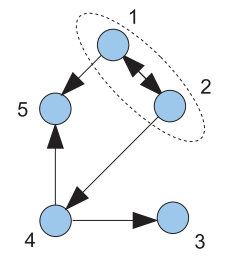

Fig. 10: Consensus graph $\mathcal{G}$ used in the simulation of the control law (67) when the consensus graph is not strongly connected, but has a closed strongly connected component.

\section{CONCLUSiOnS AND Future WORK}

Aside from the main result that consensus and shape control can be combined, the striking feature of the analysis is that
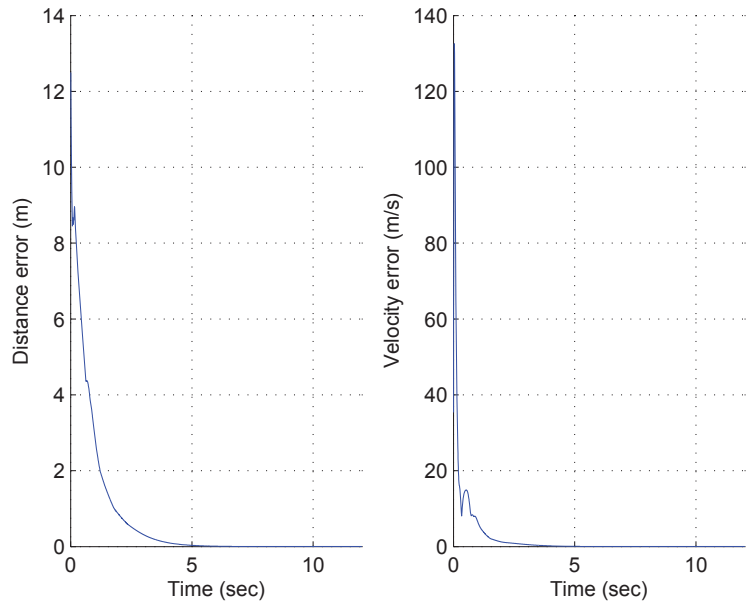

Fig. 11: The sum of the distance errors between the neighbor agents and the 2-norm of the vector of velocity errors when the system dynamics are governed by (67).

for this to occur with the controls being injected in an additive fashion, shape control has to be done with a double integrator model, as compared with the situation when no consensus is sought, and a single integrator model is used.

There are open issues not addressed here, which we now record. First, we have not recorded a result on combining consensus and shape control when the consensus graph is directed but there is no identified leader. This is actually straightforward to do when the consensus graph is strongly connected. However, since consensus alone will occur if the graph has a unique closed strongly connected component, one would expect that the issue of combining for such a graph the consensus problem with the shape control problem should be addressable. Actually, this appears quite challenging in a technical sense. Second, we note that recent work on shape control alone has shown the possibility of achieving shape control in a finite time with the aid of switching functions, [19]. Switching functions are by nature discontinuous, and accordingly existence and uniqueness questions for differential equation solutions come into play. It would be worthwhile to examine what could be done with combined consensus and shape control.

\section{APPENDIX 1}

Lemma 5: Consider $J_{F_{1}}(\hat{p}, \hat{q})$ in (34) in which $\hat{\mathcal{L}}$ is a symmetric positive definite matrix and $J_{g_{\hat{p}}}(\hat{p})^{\top} J_{g_{\hat{p}}}(\hat{p})$ is a symmetric matrix that has one zero eigenvalue and the rest are real and positive. Then $J_{F_{1}}(\hat{p}, \hat{q})$ has one zero eigenvalue and the rest have negative real parts. Furthermore, if $\nu$ is the eigenvector corresponding to the zero eigenvalue of $J_{g_{\hat{p}}}(\hat{p})^{\top} J_{g_{\hat{p}}}(\hat{p})$, then the eigenvector corresponding to the zero eigenvalue of $J_{F_{1}}(\hat{p}, \hat{q})$ is $\left[\nu^{\top} 0\right]^{\top}$.

Proof: We first show that $J_{F_{1}}(\hat{p}, \hat{q})$ has only one zero eigenvalue and the rest have negative real parts. Define $\hat{\mathcal{J}}=$ $J_{g_{\hat{p}}}(\hat{p})^{\top} J_{g_{\hat{p}}}(\hat{p})$. let $\lambda$ be an eigenvalue of $J_{F_{1}}(\hat{p}, \hat{q})$ and let 
$\left[\begin{array}{ll}v_{1}^{\top} & v_{2}^{\top}\end{array}\right]^{\top}$ be the associated eigenvector. Then $\lambda$ satisfies

$$
\left[\begin{array}{cc}
\lambda I & -I \\
\hat{\mathcal{J}} & \lambda I+\hat{\mathcal{L}}
\end{array}\right]\left[\begin{array}{l}
v_{1} \\
v_{2}
\end{array}\right]=0
$$

which can also be written as

$$
\begin{gathered}
\lambda v_{1}=v_{2} \\
\hat{\mathcal{J}} v_{1}+\lambda v_{2}+\hat{\mathcal{L}} v_{2}=0 .
\end{gathered}
$$

Substituting (74) into (75), we derive that

$$
\left(\lambda^{2} I+\lambda \hat{\mathcal{L}}+\hat{\mathcal{J}}\right) v_{1}=0
$$

and so

$$
v_{1}^{\top}\left(\lambda^{2} I+\lambda \hat{\mathcal{L}}+\hat{\mathcal{J}}\right) v_{1}=0 .
$$

The left-hand side of the above equation can also be written as

$$
\lambda^{2}\left\|v_{1}\right\|^{2}+v_{1}^{\top} \hat{\mathcal{L}} v_{1} \lambda+v_{1}^{\top} \hat{\mathcal{J}} v_{1}=0 .
$$

Denote $v_{1}^{\top} \hat{\mathcal{L}} v_{1}=a$ and $v_{1}^{\top} \hat{\mathcal{J}} v_{1}=b$. It is clear that $a>0$ for whatever nonzero $v_{1}$ as $\hat{\mathcal{L}}$ is positive definite. Similarly, we know that $b=0$ when $v_{1}$ is in the null space of $\hat{\mathcal{J}}$, which is of one dimension, and $b>0$ for all $v_{1}$ not in the null space of $\hat{\mathcal{J}}$. Therefore, rewriting (78) as

$$
\lambda^{2}\left\|v_{1}\right\|^{2}+a \lambda+b=0
$$

and solving for the roots, we obtain

$$
\lambda=\frac{-a \pm \sqrt{a^{2}-4 b\left\|v_{1}\right\|^{2}}}{2\left\|v_{1}\right\|^{2}} .
$$

As $a>0$ and $b \geq 0$ (for which $b=0$ only when $v_{1}$ is in the one dimensional null space of $\hat{\mathcal{J}}$ ), it then follows from (80) that $\lambda$ has negative real part for all $v_{1}$ not in the null space of $\hat{\mathcal{J}}$, and it gives rise to a zero eigenvalue when $v_{1}$ is in the null space of $\hat{\mathcal{J}}$.

Now assume $\lambda=0$ and therefore $v_{1}$ is in the null space of $\hat{\mathcal{J}}$. Then according to (74) and (75), the eigenvector of $J_{F_{1}}(\hat{p}, \hat{q})$ corresponding to its zero eigenvalue is $\left[\begin{array}{ll}v_{1}^{\top} & 0\end{array}\right]^{\top}$ where $\hat{\mathcal{J}} v_{1}=$ 0 .

\section{APPENDIX 2}

Proof of Lemma 2: To show that $\hat{\mathcal{M}}$ is a center manifold for (36), we need to show that $\hat{\mathcal{M}}$ is invariant and is tangent to the center subspace of (36) at the origin. The set $\hat{\mathcal{M}}$ is invariant because it is the equilibrium set of (36). Instead of showing that $\hat{\mathcal{M}}$ is tangent to the center subspace of (36) at the origin, we can show that $\hat{\Omega}_{1}$ in (31) is tangent to the eigenvector of $J_{F_{1}}(\hat{z})$ corresponding to its zero eigenvalue at the origin. As shown in Lemma 5 in Appendix 1, the eigenvector of $J_{F_{1}}(\hat{z})$ corresponding to its zero eigenvalue is $\left[\begin{array}{ll}\nu_{1}^{\top} & 0\end{array}\right]^{\top}$ where $\nu_{1}$ satisfies $J_{g_{\hat{p}}}(\hat{p})^{\top} J_{g_{\hat{p}}}(\hat{p}) \nu_{1}=0$. Rewrite $\hat{\Omega}_{1}$ as

$$
\hat{\Omega}_{1}=\left\{(\hat{p}, \hat{q}): \hat{G}(\hat{p}, \hat{q}):=\left[\begin{array}{c}
g_{\hat{p}}(\hat{p})-\mathrm{d}^{*} \\
\hat{q}
\end{array}\right]=0\right\} .
$$

Then the Jacobian of $\hat{G}(\hat{p}, \hat{q})$ is

$$
J_{\hat{G}}(\hat{z})=\left[\begin{array}{cc}
J_{g_{\hat{p}}}(\hat{p}) & 0 \\
0 & I
\end{array}\right] \text {. }
$$

In order to show that $\hat{\Omega}_{1}$ is tangent to $\left[\begin{array}{ll}\nu_{1}^{\top} & 0\end{array}\right]^{\top}$ at the origin, we show that $J_{\hat{G}}(0)\left[\begin{array}{ll}\nu_{1}^{\top} & 0\end{array}\right]^{\top}=0$ :

$$
J_{\hat{G}}(0)\left[\begin{array}{c}
\nu_{1} \\
0
\end{array}\right]=\left[\begin{array}{cc}
J_{g_{\hat{p}}}(0) & 0 \\
0 & I
\end{array}\right]\left[\begin{array}{c}
\nu_{1} \\
0
\end{array}\right]=\left[\begin{array}{c}
J_{g_{\hat{p}}}(0) \nu_{1} \\
0
\end{array}\right] .
$$

We want to show that $J_{g_{\hat{p}}}(0) \nu_{1}=0$. This comes from $\left\|J_{g_{\hat{p}}}(0) \nu_{1}\right\|^{2}=\nu_{1}^{\top} J_{g_{\hat{p}}}(0)^{\top} J_{g_{\hat{p}}}(0) \nu_{1}$ and that $\nu_{1}$ is the eigenvector of $J_{g_{\hat{p}}}(0)^{\top} J_{g_{\hat{p}}}(0)$ corresponding to its zero eigenvalue. Hence $\nu_{1}^{\top} J_{g_{\hat{p}}}(0)^{\top} J_{g_{\hat{p}}}(0) \nu_{1}$ is zero and therefore $\hat{\Omega}_{1}$ is tangent to $\left[\begin{array}{ll}\nu_{1}^{\top} & 0\end{array}\right]^{\top}$ at the origin.

\section{REFERENCES}

[1] B. D. O. Anderson, Z. Lin, and M. Deghat. "Combining distance-based formation shape control with formation translation," Developments in Control Theory Towards Glocal Control, pp. 121-130, 2012.

[2] L. Krick, M. E. Broucke, and B. A. Francis. "Stabilization of infinitesimally rigid formations of multi-robot networks," International Journal of Control, vol. 82, pp. 423-439, 2009.

[3] Z. Lin, Distributed Control and Analysis of Coupled Cell Systems. VDM Verlag Dr Müller, 2008.

[4] W. Ren, R. W. Beard, and E. M. Atkins. "Information Consensus in Multivehicle Cooperative Control," IEEE Control Systems Magazine, vol. 27, no. 2, pp. 71-82, 2007.

[5] R. Olfati-Saber, "Flocking for Multi-Agent Dynamic Systems: Algorithms and Theory," IEEE Trans. Autom. Control, vol. 51, no. 3, pp. 401-420, March 2006.

[6] H. G. Tanner, A. Jadbabaie, and G. J. Pappas, "Flocking in Fixed and Switching Networks," IEEE Trans. Autom. Control, vol. 52, no. 5, pp. 863-868, May 2007.

[7] D. V. Dimarogonas and K. H. Johansson, "On the stability of distancebased formation control," in Proc. 47th IEEE Conf. Decision and Control, 2008, pp. 1200-1205.

[8] H. Su, X. Wang, and Z. Lin, "Flocking of Multi-Agents With a Virtual Leader," IEEE Trans. Autom. Control, vol. 54, no. 2, pp. 293-307, February 2009.

[9] T. H. Summers, C. Yu, S. Dasgupta, and B. D. O. Anderson, "Contro of minimally persistent leader-remote-follower and coleader formations in the plane," IEEE Transactions on Automatic Control, vol. 56, no. 12 pp. 2778-2792, 2011

[10] W. Ren and E. Atkins, "Distributed multi-vehicle coordinated control via local information exchange," International Journal of Robust and Nonlinear Control, vol. 17, no. 10-11, pp. 1002-1033, 2007.

[11] Y. Cao, W. Ren and Z. Meng, "Decentralized finite-time sliding mode estimators and their applications in decentralized finite-time formation tracking," Systems \& Control Letters, vol. 59, no. 9, pp. 522-529, 2010.

[12] B. D. O. Anderson, C. Yu, B. Fidan, and J. M. Hendrickx, "Rigid graph control architectures for autonomous formations," IEEE Control Systems Magazine, vol. 28, no. 6, pp. 48-63, 2008.

[13] T. Tay and W. Whiteley, "Generating isostatic frameworks," Structural Topology, vol. 11, no. 1, pp. 2169, 1985.

[14] D. V. Dimarogonas and K. H. Johansson, "Bounded control of network connectivitiy in multi-agent systems," IET Control Theory and Applications, vol. 4, pp. 1330-1338, 2010.

[15] J. Carr, Applications of centre manifold theory, Springer-Verlag, 1981.

[16] S. Sastry, Nonlinear systems: analysis, stability, and control, Springer, 1999.

[17] R. A. Horn and C. R. Johnson, Matrix analysis, Cambridge University Press, 1985.

[18] C. D. Godsil and G. Royle, Algebraic graph theory, Springer New York, 2001.

[19] F. Xiao, L. Wang, J. Chen and Y. Gao, "Finite-time formation control for multi-agent system," Automatica, vol. 45, pp. 2605-2611, 2009. 